\title{
Kernos
}

Revue internationale et pluridisciplinaire de religion grecque antique

$28 \mid 2015$

Varia

\section{Revue des ouvrages reçus}

\section{(2) OpenEdition}

\section{Journals}

Édition électronique

URL : http://journals.openedition.org/kernos/2370

DOI : 10.4000/kernos.2370

ISSN : 2034-7871

\section{Éditeur}

Centre international d'étude de la religion grecque antique

\section{Édition imprimée}

Date de publication : 1 octobre 2015

Pagination : 306-308

ISBN : 978-2-87562-055-2

ISSN : 0776-3824

Référence électronique

"Revue des ouvrages reçus », Kernos [En ligne], 28 | 2015, mis en ligne le 10 octobre 2015, consulté le 24 septembre 2020. URL : http://journals.openedition.org/kernos/2370 ; DOI : https://doi.org/ $10.4000 /$ kernos. 2370

Ce document a été généré automatiquement le 24 septembre 2020 


\section{Revue des ouvrages reçus}

1 ALROTH Brita, SCHEFFER Charlotte, Attitutes towards the Past in Antiquity. Creating Identities. Proceedings of an International Conference held at Stockholm University, 15-17 May 2009, Stockholm, Acta Universitatis Stockholmiensis, 2013. 1 vol. $19 \times 26,5 \mathrm{~cm}, 325 \mathrm{p}$. (Stockholm Studies in Classical Archaeology, 14). ISBN : 978-9-187-23547-4.

2 BADOUD Nathan, Le Temps de Rhodes. Une chronologie des inscriptions de la cité fondée sur l'étude de ses institutions, München, C.H. Beck, 2015. 1 vol. 21,5×30 cm, xviii+542 p. (Vestigia. Beiträge zur Alten Geschichte, 63). ISBN : 978-3-406-64035-3

3 BARbu Daniel, BORgeaud Philippe, LOZAT Mélanie, MEYlan Nicolas, RENDU LoISEl AnneCaroline (éd.), MASSA Francesco (coll.), Le savoir des religions. Fragments d'historiographie religieuse, Gollion, Infolio, 2014. 1 vol. $14,5 \times 21,5 \mathrm{~cm}, 552 \mathrm{p}$. (Asdiwal. Revue genevoise d'anthropologie et d'histoire des religions, suppl. 2). ISBN : 978-2-88474-814-8. BLouIN Katherine, Triangular Landscapes: Environment, Society, and the State in the Nile Delta under Roman Rule, Oxford, Oxford University Press, 2014. 1 vol. 14,5 × $22 \mathrm{~cm}$, xxvi+430 p. (Oxford Studies on the Roman Economy). ISBN : 978-0-19-968872-2.

5 BRICAULt Laurent, VEYMIERS Richard (éd.), Bibliotheca Isiaca III, Bordeaux, Ausonius Éditions, 2014. 1 vol. $21 \times 29,5 \mathrm{~cm}, 485$ p. ISBN : 978-2-356-13121-8.

6 BRULÉ Pierre, Les sens du poil (grec), Paris, Les Belles Lettres, 2015. 1 vol. $15 \times 21,5 \mathrm{~cm}$, 569 p. ISBN : 978-2-251-44534-2.

7 CALAME Claude, Qu'est-ce que la mythologie grecque?, Paris, Éditions Gallimard, 2015. 1 vol. $11 \times 18 \mathrm{~cm}, 732$ p. (Collection Folio Essai). ISBN : 978-2-07-044578-3.

8 CAMPBell Gordon L. (éd.), The Oxford Handbook of Animals in Classical Thought and Life, Oxford, Oxford University Press, 2014. 1 vol. $18 \times 25,5 \mathrm{~cm}$. ix $+633 \mathrm{p}$. ISBN : 978-0-19-958942-5.

9 DE JONG Irene J.F., Narratology \& Classics. A Practical Guide, Oxford University Press, 2014. 1 vol. $14 \times 21,5 \mathrm{~cm}$, viii+230 p. ISBN : 978-0-19-968870-8.

10 Dialogues d'histoire ancienne, 41/1 (2015), Besançon, Presses universitaires de FrancheComté, 2015.1 vol. $16 \times 22 \mathrm{~cm}, 401$ p. ISSN : 0755-7256. 
ENGELS David, VAN NUfFELEN Peter (éd.), Religion and Competition in Antiquity, Bruxelles, Éditions Latomus, 2014. 1 vol. $16 \times 24 \mathrm{~cm}, 307$ p. (Collection Latomus, 343). ISBN : 978-2-87031-290-3.

ESTIENNE Sylvia, HUET Valérie, LISSARRAGUE François, PROST Francis (éd.), Figures de dieux. Construire le divin en images, Rennes, Presses universitaires, 2014. 1 vol. 15,5 $24 \mathrm{~cm}$, 380 p. (Collection « Histoire »). ISBN : 978-2-7535-3522-0.

FONTANA Federica, MURGIA Emanuela (éd.), Sacrum facere. Atti del II Seminario di Archeologia del Sacro. Contaminazioni: forme di contatto, traduzione e mediazione nei sacra del mondo greco e romano, Trieste, 19-20 aprile 2013, Edizioni Università di Trieste, 2014. 1 vol. $17 \times 24 \mathrm{~cm}$, viii+332 p. (POLYMNIA. Studi di archeologia, 6). ISBN : 978-88-8303-576-0.

FRANCHI Elena, PROIETTI Giorgia (éd.), Guerra e memoria nel mondo antico, Trento, Università degli Studi di Trento - Dipartimento di Lettere e Filosofia, 2014. 1 vol. $15,5 \times 21,5 \mathrm{~cm}, 361$ p. (Quaderni, 6). ISBN : 978-8-884-43589-7.

Gaia. Revue interdisciplinaire sur la Grèce archaïque, 17 (2015), Grenoble, ERGA (Université Stendhal - Grenoble 3), 2015.1 vol. $16 \times 24$ cm, 283 p. ISSN : 1262-3717.

GHERCHANOC Florence (éd.), L'histoire du corps dans l'Antiquité: bilan historiographique. Journée de printemps de la SOPHAU du 25 mai 2013, Besançon, Presses universitaires de Franche-Comté, 2015. 1 vol. $16 \times 22 \mathrm{~cm}, 196$ p. (Dialogues d'histoire ancienne, suppl. 14). ISBN : 978-2-848-67526-8.

7 GNOLI Tommaso, muccioli Federicomaria (a cura di), Divinizzazione, culto del sovrano $e$ apoteosi tra Antichità e Medioevo, Bologna, Bononia University Press, 2014. 1 vol. $17 \times 24 \mathrm{~cm}, 534$ p. (Storia antica). ISBN : 978-88-7395-912-0.

GUARISCo Diana, Santuari "gemelli" di una divinità. Artemide in Attica, Bologna, Bononia University Press, 2015. 1 vol. $17 \times 24 \mathrm{~cm}, 162$ p. (Dipartimento storia culture civiltà, Sezione Storia antica, 2). ISBN : 978-8-869-23022-6.

9 HADOT Ilsetraut, Athenian and Alexandrian Neoplatonism and the Harmonization of Aristotle and Plato, traduit par Michael Chase, Leiden/Boston, Brill, 2015. 1 vol. $16 \times 24 \mathrm{~cm}$, $\mathrm{X}+188$ p. (Studies in Platonism, Neoplatonism, and the Platonic Tradition, 18). ISBN : 978-9-004-28007-6.

20 HOLlinSHEAD Mary B., Shaping Ceremony. Monumental Steps and Greek Architecture, Madison, The University of Wisconsin Press, 2015. 1 vol. $21 \times 26 \mathrm{~cm}, x i v+233$ p. ISBN : 978-0-29-9301101.

21 HORNBLOWER Simon, Lykophron: Alexandra. Greek Text, Translation, Commentary, \& Introduction, Oxford, Oxford University Press, 2015. 1 vol. $16 \times 24 \mathrm{~cm}$. ISBN : 978-0-199-57670-8.

ISLER-KERÉNYI Cornelia, Dionysos in Classical Athens. An Understanding through Images, traduit par Anna Beerens, Leiden/Boston, Brill, 2015. 1 vol. $16 \times 24 \mathrm{~cm}, \mathrm{xx}+290 \mathrm{p}$. (Religions in the Graeco-Roman World, 181). ISBN : 987-9-004-27011-4.

KERÉNYI Károly, BRELICH Angelo, Tra gli asfodeli dell'Elisio. Carteggio 1935-1959, éd. par Andrea Alessandri, traduit par Andrea Nagy, Roma, Editori Riuniti university press, 2011. 1 vol. 15 × $21 \mathrm{~cm}, 373$ p. ISBN : 978-8-864-73064-6.

LÉVI Nicolas, La révélation finale à Rome: Cicéron, Ovide, Apulée. Étude sur le "Songe de Scipion " (De republica, VI), le discours de Pythagore (Métamorphoses, XV), et la théophanie 
d'Isis ( Métamorphoses, XI), Paris, Presses de l'université Paris-Sorbonne, 2014. 1 vol. 16 $\times 24 \mathrm{~cm} .537$ p. ISBN : 978-2-84050-945-5. LINCOLN Bruce, Politique du paradis. Religion et empire en Perse achéménide, Genève, Labor et Fides, 2015. 1 vol. 15 × 22,5 cm, 143 p. (Histoire des Religions). ISBN : 978-2-830-91570-9.

MILI Maria, Religion and Society in Ancient Thessaly, Oxford University Press, 2015. 1 vol. $16 \times 24 \mathrm{~cm}$, xiv+430 p. (Oxford Classical Monographs). ISBN : 978-0-19-871801-7.

MORENo Alfonso, thomas Rosalind (éd.), Patterns of the Past. Epitēdeumata in the Greek Tradition, Oxford University Press, 2014. 1 vol. $14,5 \times 22,5 \mathrm{~cm}$, viii +267 p. ISBN : 978-0-19-966888-5.

MORETTI Jean-Claude (éd.), Le sanctuaire de Claros et son oracle. Actes du colloque international de Lyon, 13-14 janvier 2012, Lyon, Maison de l'Orient et de la Méditerranée, 2014. 1 vol. $21 \times 29,5 \mathrm{~cm}, 258$ p. (Travaux de la Maison de l'Orient et de la Méditerranée, 65). ISBN : 978-2-35668-047-1.

NIELSEN Inge, Housing the Chosen. The Architectural Context of Mystery Groups and Religious Associations in the Ancient World, Turnhout, Brepols, 2014. 1 vol. $21,5 \times 30 \mathrm{~cm}, 322 \mathrm{p}$. (Contextualizing the Sacred, 2) ISBN : 978-2-503-54437-3.

Philotheos. International for Philosophy and Theology, 14 (2014), Beograd, 2014. 1 vol. $17 \times 24 \mathrm{~cm}, 384$ p. ISSN : 1451-3455.

RICHARDSON James H., SANTANGELO Federico (éd.), The Roman Historical Tradition. Regal and Republican Rome, Oxford University Press, 2014. 1 vol. $13,5 \times 21,5 \mathrm{~cm}, \mathrm{x}+372$ p. (Oxford Readings in Classical Studies). ISBN : 978-0-19-965785-8.

RÜPKE Jörk, From Jupiter to Christ: On the History of Religion in the Roman Imperial Period, Oxford, Oxford University Press, 2014. 1 vol. $14,5 \times 22 \mathrm{~cm}$, viii +328 p. ISBN : 978-0-19-870372-3.

SCANLON Thomas F. (ed.), Sport in the Greek and Roman Worlds. Volume 1: Early Greece, the Olympics, and Contests, Oxford, Oxford University Press, 2014. 1 vol. $14 \times 21 \mathrm{~cm}$, xii+338 p. (Oxford Readings in Classical Studies). ISBN : 978-0-19-921532-4.

SCANLON Thomas F. (ed.), Sport in the Greek and Roman Worlds. Volume 2: Greek Athletic Identities and Roman Sports and Spectacle, Oxford, Oxford University Press, 2014. 1 vol. $14 \times 21 \mathrm{~cm}$, xii+390 p. (Oxford Readings in Classical Studies). ISBN : 978-0-19-870378-5.

SCHEID John, SVENBRO Jesper, La tortue et la lyre. Dans l'atelier du mythe antique, Paris, CNRS Éditions, 2014. 1 vol. $14 \times 22 \mathrm{~cm}, 229$ p. ISBN : 978-2-271-07883-4.

SOREL Reynal, Dictionnaire du paganisme grec. Notions et débats autour de l'époque classique, Paris, Les Belles Lettres, Paris, 2015. 1 vol. $15 \times 21,5$ cm, 513 p. (Vérité des Mythes).

STEINHAUER Julietta, Religious Associations in the Post-Classical Polis, Stuttgart, Franz Steiner Verlag, 2014. 1 vol. $17 \times 24 \mathrm{~cm}, 189$ p. (Postdamer Altertumswissenschaftliche Beiträge, 50). ISBN : 978-3-515-10646-7.

TORTORELli GHIDINI Marisa (éd.), Aurum. Funzioni e simbologie dell'oro nelle culture del Mediterraneo antico, Rome, « L'Erma » di Bretschneider, 2014. 1 vol. $17 \times 24 \mathrm{~cm}, 436$ p.+ill. (Studia Archaeologica, 193). ISBN : 978-88-913-0482-7.

TRAMPEDACH Kai, Politische Mantik. Die Kommunikation über Götterzeichen und Orakel im klassischen Griechenland, Heidelberg, Verlag Antike, 2015. 1 vol. $16 \times 22,5 \mathrm{~cm}, 650 \mathrm{p}$. (Studien zur Alten Geschichte, 21). ISBN : 978-3-938-03278-7. 
40 Oxford University Press, 2014. 1 vol. $14,5 \times 22 \mathrm{~cm}$, xviii +420 p. (Classical Presences). ISBN : 978-0-19-967275-2.

41 VAHTIKARI Vesa, Tragedy Performances outside Athens in the Late Fifth and the Fourth Centuries BC, Helsinki, Finnish Institute in Athens, 2014. 1 vol. 17,5 × $25 \mathrm{~cm}, 334$ p.+ill. (Papers and Monographs of the Finnish Institute at Athens, 20). ISBN 978-9-526-72118-7.

42 VAN STEEN Gonda, Stage of Emergency. Theater and Public Performance under the Greek Military Dictatorship of 1967-1974, Oxford, Oxford University Press, 2015. 1 vol. $14,5 \times 22,5 \mathrm{~cm}, \mathrm{xviii}+376$ p. (Classical Presences). ISBN : 978-0-19-871832-1.

43 WARDLE David, Suetonius, Life of Augustus, translated with introduction and historical commentary, Oxford, Oxford University Press, 2014. 1 vol. $16 \times 23,5 \mathrm{~cm}, \mathrm{x}+603 \mathrm{p}$. (Clarendon Ancient History Series). ISBN : 978-0-19-968646-9.

44 WEST M.L., The Making of the Odyssey, Oxford, Oxford University Press, 2014. 1 vol. $14,5 \times 22,5 \mathrm{~cm}, \mathrm{xii}+315$ p. ISBN : 978-0-19-871836-9.

45 WIJMA Sara M., Embracing the Immigrant. The Participation of Metics in Athenian Polis Religion $\left(5^{\text {th }}-4^{\text {th }}\right.$ century BC), Stuttgart, Franz Steiner Verlag, 2014. 1 vol. $17,5 \times 24,5 \mathrm{~cm}, 197 \mathrm{p}$. (Historia - Einzelschriften, 233). ISBN : 978-3-515-10642-9. 